\title{
IMAGE ANALYSIS OF OLFACTORY RESPONSES IN THE PROCEREBRUM OF THE TERRESTRIAL SLUG LIMAX MARGINATUS
}

\author{
SHO'ICHI TODA, SHIGENORI KAWAHARA* AND YUTAKA KIRINO \\ Laboratory of Neurobiophysics, School of Pharmaceutical Sciences, The University of Tokyo, 7-3-1 Hongo, \\ Bunkyo-ku, Tokyo 113-0033, Japan \\ *Author for correspondence (e-mail: kawahara@mol.f.u-tokyo.ac.jp)
}

Accepted 7 July; published on WWW 7 September 2000

\begin{abstract}
Summary
Neural oscillations have been found to occur in the olfactory centers of some vertebrates and invertebrates, including the procerebrum of the terrestrial slug Limax marginatus. Using optical recording with the potential-sensitive dye di-4-ANEPPS, we analyzed the spatiotemporal pattern of procerebral neural activities in response to odorants applied to an in vitro brain-superior tentacle preparation. The odor of rat chow, on which the slugs were normally fed, increased the frequency of the oscillation. Garlic odor, which induced aversive behavior in the slug, caused a transient increase in oscillation

parallel with modulation of the frequency. The cycle-bycycle average of the optical signals showed that a large area of the cerebral ganglia, including the procerebrum, was depolarized during the initial increase in frequency. During the second increase, however, the net depolarization was most prominent in the terminal mass of the procerebrum. These results suggest that the level of depolarization generated by interactions among the neurites projecting to the terminal mass, such as the neurites of the nonbursting neurons, may control neural oscillations in the procerebrum.
\end{abstract} frequency during stimulation, followed by a second increase in oscillation frequency when the stimulus was terminated. Wave propagation from the distal to the proximal region of the procerebrum was accelerated in

Key words: slug, Limax marginatus, neural oscillation, olfaction, odour, optical recording, procerebrum.

\section{Introduction}

Oscillatory activity is found in neural networks of rhythmic motor systems (Grillner et al., 1995; Brierley et al., 1997) and in a variety of other brain regions in vertebrates and invertebrates (Laurent and Naraghi, 1994; Ritz and Sejnowski, 1997; Castelo-Branco et al., 1998). The neural oscillations that occur in rhythmic motor systems are straightforward to interpret because they are directly coupled to motor functions that are retained in isolated preparations from both vertebrates and invertebrates. This feature means that motor systems have proved valuable for studying the mechanisms and roles of neural oscillations, including their effects on sensory information processing (Kawahara et al., 1994; Degtyarenko et al., 1996; Vinay et al., 1996). In contrast, neural oscillations in other brain regions that process sensory information have not been well studied because it is difficult to elucidate the functional significance and mechanism of oscillations in mammalian sensory systems that lose their functions in vitro.

Recent studies have revealed neural oscillations in the olfactory systems of terrestrial slugs (Gelperin and Tank, 1990; Kawahara et al., 1997) and insects (Laurent and Naraghi, 1994) that might be functionally analogous to those expressed in the mammalian olfactory bulb (Freeman, 1978). Since the central nervous systems of invertebrates, together with the sensory organs and attached muscular systems, preserve their functions well even after isolation from the organism, they should serve as a good in vitro model system for studying the role and mechanism of oscillatory activity in the neural networks that process sensory information.

The terrestrial slug possesses a high capacity for odor-taste associative learning (Sahley et al., 1981; Gelperin et al., 1985; Yamada et al., 1992; Sekiguchi et al., 1997). The procerebrum, which is located at the tip of the cerebral ganglion, is known to be the olfactory center in slugs and snails (Chase, 1985; Gelperin and Tank, 1990) and is likely to be the site of memory formation and consolidation (Kimura et al., 1998a,b). Recent optical image analyses using a potential-sensitive dye and field potential recordings have revealed that procerebral interneurons undergo oscillatory activity at a frequency of approximately $0.7 \mathrm{~Hz}$ (Delaney et al., 1994; Kleinfeld et al., 1994; Kawahara et al., 1997; Kimura et al., 1998b). These oscillations can be modulated by an odor stimulus either to the superior tentacular nose (Gervais et al., 1996) or to the inferior tentacular nose (Kimura et al., 1998a). However, the details and mechanism of frequency modulation have yet to be investigated. 


\section{S. TODA, S. KAWAHARA AND Y. KIRINO}

We found that spontaneous fluctuations in the frequency of oscillation in the procerebrum of the slug Incilaria bilineata were correlated with changes in the mean membrane potential measured optically in the terminal mass (Kawahara et al., 1997). This suggests the possibility that modulation of the frequency of the oscillation may be related to neural activity in the terminal mass. The superior tentacle nerve, which projects into the terminal mass, exhibits an oscillation of the optical signal in synchrony with the procerebral oscillation (Kawahara et al., 1997), suggesting that there are local feedback circuits that could furnish active properties to the terminal mass. In this paper, we have examined modulation of the frequencies of neural oscillations in the procerebrum of Limax marginatus by odor stimulation of the superior tentacular nose, and have found that net depolarization in the terminal mass occurs in parallel with an increase in the frequency of the oscillation and in the velocity of wave propagation.

\section{Materials and methods}

Slugs Limax marginatus (Müller) were bred from eggs in our laboratory (Kawahara et al., 1997). They were deprived of food for several days and then tested for their response to their normal daily food, rat chow. Each slug (weighing 1-2 g) was placed on a glass plate and presented with rat chow moistened with distilled water. Only those slugs that approached the rat chow and showed signs of feeding were selected and used in subsequent experiments. Slugs were anesthetized by injecting $0.2-0.5 \mathrm{ml}$ of isotonic $\mathrm{Mg}^{2+}$ solution (in $\mathrm{mmoll}^{-1}: \mathrm{MgCl}_{2}$, 57.6; glucose, 5.0; and Hepes, 5.0; pH7.6 adjusted with $\mathrm{NaOH}$ ) into the body cavity. The right and left cerebral ganglia with the superior tentacles attached were isolated and placed in a chamber with two compartments, one for the tentacle and the other for the ganglion, both filled with saline of the following composition (in $\mathrm{mmoll}^{-1}$ ): $\mathrm{NaCl}, 70.0 ; \mathrm{KCl}, 2.0$; $\mathrm{CaCl}_{2}$, 4.9; $\mathrm{MgCl}_{2}$, 4.7; glucose, 5.0; and Hepes, 5.0; $\mathrm{pH} 7.6$ adjusted with $\mathrm{NaOH}$. Only one of the superior tentacular noses was placed in the tentacle compartment and used later for odor stimulation. The other nose and the cerebral ganglia were placed in the ganglion compartment and stained with a saturated solution (approximately $0.02 \%$, w/v) of di-4ANEPPS (Molecular Probes, Eugene, OR, USA) for $20-30 \mathrm{~min}$ at $20^{\circ} \mathrm{C}$. After washing with saline, they were covered with a nylon mesh and then immobilized with $4 \%$ low-melting-point agarose (Sigma, St Louis, MO, USA).

The equipment for optical recording was the same as that described by Kawahara et al. (1997). The optical signals were recorded using a $128 \times 128$ pixel MOS sensor camera (HR Deltaron 1700; Fuji Photo Film Co., Tokyo, Japan) attached to the bottom TV port of an inverted fluorescence microscope (IX70; Olympus, Tokyo, Japan) equipped with a 10× objective lens (NA 0.40), a band-pass excitation filter $(530-550 \mathrm{~nm})$, a dichroic mirror $(565 \mathrm{~nm})$ and a long-pass emission filter $(580 \mathrm{~nm})$. A $100 \mathrm{~W}$ halogen lamp with a direct current power supply was used as the light source. To generate an image, the optical signals were accumulated for $38.4 \mathrm{~ms}$. The camera system was designed to measure the difference between the experimental fluorescence intensity and the initial fluorescence intensity stored before the experimental measurement was made. The original values of optical signals were recovered from the initial value and the difference values. The optical data collected were rebinned to $64 \times 64$ pixels and analyzed as in our previous study (Kawahara et al., 1997), except that the data acquired during and just after odor stimulation were eliminated from the calculation of the linear regression line used for the correction for photobleaching. Additional spatial or temporal filters were not used for image analysis, unless indicated otherwise. The value of the fractional change in the intensity of fluorescence $(\Delta F / F)$ of each pixel indicates the relative polarization to the time-averaged level of the membrane potential of neurons in the $13 \mu \mathrm{m} \times 13 \mu \mathrm{m}$ area covered by the binned pixel $(2 \times 2$ original pixels $)$.

In Fig. $6, \Delta F / F$ of each pixel was averaged cycle-by-cycle to reduce the influence of the oscillation of the membrane potential as follows. First, the peaks of the depolarization were detected from $\Delta F / F$ values of $5 \times 5$ pixels in the distal region of the procerebrum (indicated by a square in the last frame in Fig. 6A). Then, the $\Delta F / F$ value of each pixel was averaged temporally between these peaks, producing successive images averaged over a cycle.

The local field potential of the procerebrum was recorded extracellularly from the surface with a glass electrode (tip diameter $20 \mu \mathrm{m}$ ) filled with saline, band-pass-filtered between 0.5 and $30 \mathrm{~Hz}$, and fed into a personal computer (Epson, Nagano, Japan) at a sampling rate of $100 \mathrm{~Hz}$.

Before odor application, the superior tentacular nose was exposed to the air by lowering the surface level of the saline in the tentacle compartment. Deodorized and humidified air was supplied at a flow rate of $0.5 \mathrm{ml} \mathrm{s}^{-1}$ through a glass pipette sited just over the nose, as described by Kimura et al. (1998b). The odor was applied to the nose for $15 \mathrm{~s}$ by switching the airflow pathway using electronic valves from a glass test tube containing a strip of filter paper soaked with distilled water to one containing a strip of filter paper soaked with either $10 \%$ rat chow or commercially available garlic paste. In this study, the responses of seven preparations, which were stimulated with both rat chow and garlic odors, were analyzed. More experiments using only one odor stimulus were performed, which support the results shown, but they are not included in the analysis.

The phototoxic effects of di-4-ANEPPS (Schaffer et al., 1994) on the odor response were examined in six preparations, which were stimulated with rat chow odor under illumination with excitation light and without the illumination. In four of the six preparations, the increase in frequency of the local field potential oscillation in response to the odor was almost identical whether the excitation light was on and off. In the other two preparations, the response under illumination was smaller than that without illumination. One of these two preparations was further tested $30 \mathrm{~min}$ after exposure to light and showed a recovery of the response. Although these results 

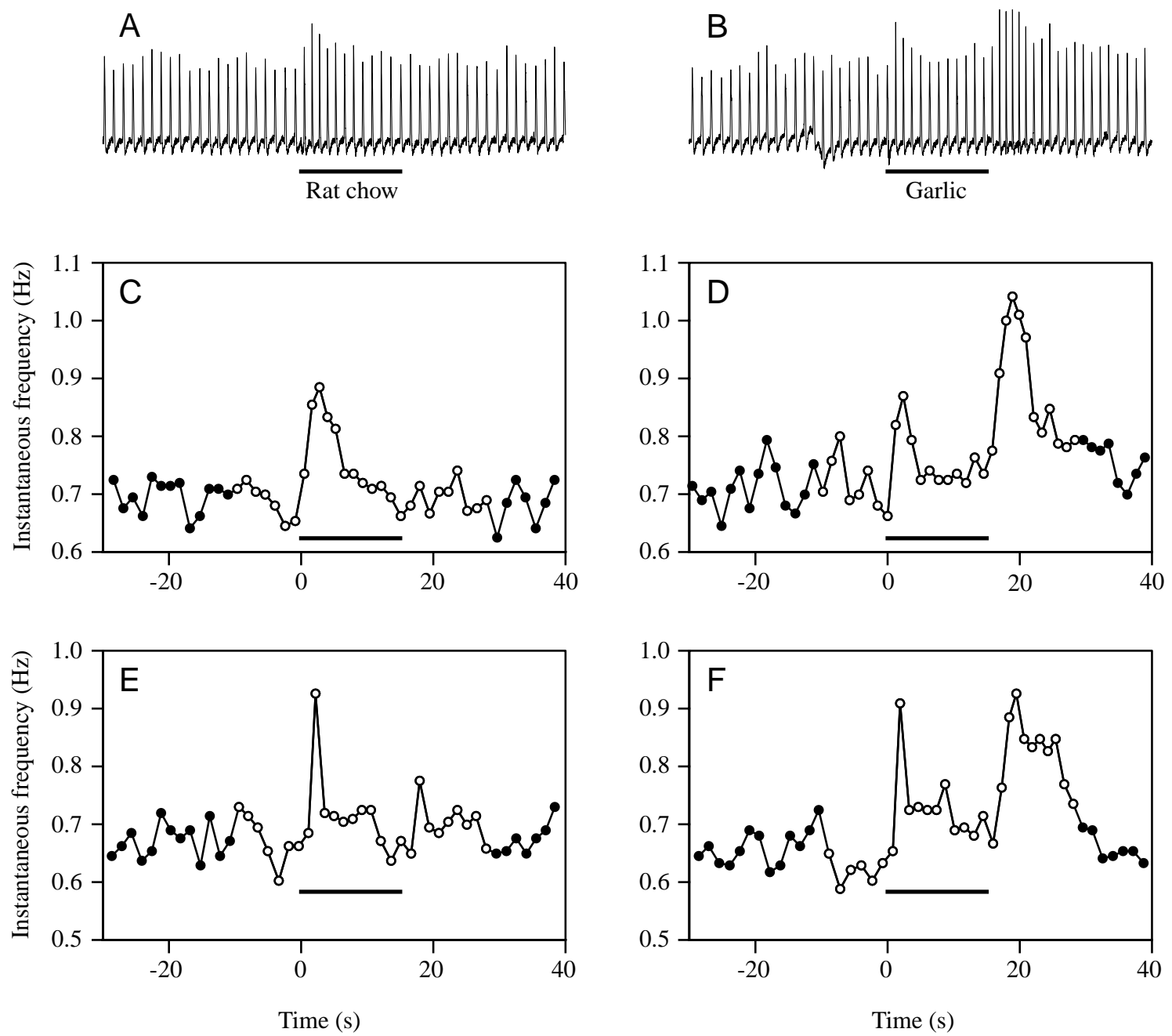

Fig. 1. Effects of odor stimulation on the oscillation of the local field potential. (A,C,E) Rat chow odor; (B,D,F) garlic odor. (A,B) The local field potential recorded from the cell layer of the procerebrum. $(\mathrm{C}-\mathrm{F})$ The instantaneous frequency of the local field potential defined as the inverse of the interval between the peaks of the local field potential. $\mathrm{C}$ corresponds to $\mathrm{A}$, and $\mathrm{D}$ corresponds to $\mathrm{B}$. The time scale is identical in A-F. Odor was applied to the superior tentacular nose ipsilateral to the recorded procerebrum for the period indicated by the bar. The sequence of application of the odors was as follows: garlic (D), rat chow (C), rat chow (E), garlic (F), each separated by approximately 10 min. The optical signal was recorded for approximately $40 \mathrm{~s}$ as indicated by the open circles in $\mathrm{C}-\mathrm{F}$.

suggested that di-4-ANEPPS had some phototoxic effect on the odor response, it was reversible and did not seem to be serious as long as an excitation light of minimum intensity was used. In fact, most of the preparations, including those that were stimulated with only one odor, showed a good response to the olfactory stimulation during optical recording.

\section{Results}

\section{Modulation of oscillatory frequency by odor stimuli}

To investigate the effects of odor stimuli on oscillatory activity in the procerebrum, isolated cerebral ganglia with the superior tentacular noses attached were stained with di-4ANEPPS, and changes in neural activity in the cerebral ganglion ipsilateral to the stimulated tentacular nose were optically recorded. Electrophysiological measurement of the local field potential in the procerebrum was also carried out on the same preparation to evaluate the effects of the odors on the frequency of oscillatory activity. Rat chow and garlic were used as odor sources. Rat chow was the normal daily food of the slugs, while the odor of garlic caused the slugs to make an avoidance withdrawal. Odors were applied to the superior nose more than twice in seven preparations.

Fig. 1 shows an example of the responses of a preparation to which odors were applied in the sequence garlic, rat chow, rat chow, garlic, each application separated by approximately $10 \mathrm{~min}$. The first trial (garlic) is shown in Fig. 1B,D, and the second trial (rat chow) is shown in Fig. 1A,C. Fig. 1E shows the third (rat chow) trial, and Fig. 1F shows the fourth (garlic) trial. When the superior tentacular nose was stimulated with the odor of rat chow, the time intervals between the peaks of the local field potential were shortened (Fig. 1A). Fig. 1C is a plot of the instantaneous frequency of the local field potential, defined as the inverse of the interval between the peaks. In this preparation, 

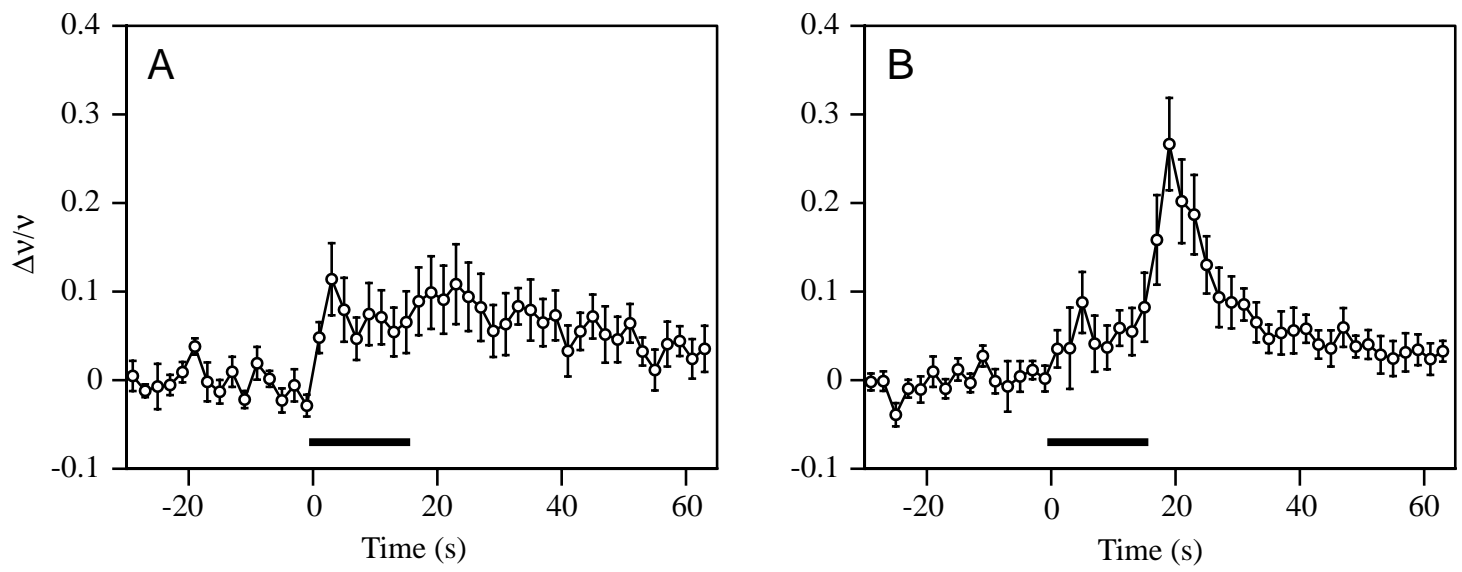

Fig. 2. Averaged data showing the effects of odor application on the frequency of the oscillation of the local field potential in different preparations. (A) Rat chow (nine trials in seven preparations); (B) garlic (10 trials in seven preparations). The ordinate shows the relative change in the instantaneous frequency normalized to the mean frequency before odor application $(\Delta v / v)$. The data from different preparations were binned into $2 \mathrm{~s}$ intervals and averaged. Error bars show the standard errors of the mean (s.E.M.). The duration of odor application is indicated by a bar.

the frequency was increased from 0.7 to $0.9 \mathrm{~Hz}$ by the odor stimulus. When the odor of garlic was applied to the nose in the same preparation, an off-response was observed in addition to the initial increase in frequency (Fig. 1B,D): the instantaneous frequency increased from 0.7 to $0.9 \mathrm{~Hz}$ upon application of the odor, and then decreased to $0.7 \mathrm{~Hz}$, producing the first peak. Just after the odor stimulus was removed, the instantaneous frequency increased again to above $1.0 \mathrm{~Hz}$, to give a second peak. Similar responses were observed with a second application of the odor stimuli (Fig. 1E, F), as well as in other preparations.

Fig. 2 summarizes the changes in the instantaneous frequency of preparations stimulated with the odor of rat chow (Fig. 2A) or garlic (Fig. 2B). Frequency was normalized for each preparation to the mean frequency over the period before odor stimulation and binned into $2 \mathrm{~s}$ intervals. The frequency before odor stimulation was $0.62 \pm 0.13 \mathrm{~Hz}$ for the rat chow odor (mean \pm S.D.; nine trials in seven preparations) and $0.64 \pm 0.12 \mathrm{~Hz}$ for the garlic odor (mean \pm S.D.; 10 trials in seven preparations). Accumulated data from different preparations clearly showed that the response to the garlic odor had an offresponse that was larger than the first peak, while the response to the rat chow odor had a small off-response. Statistical analysis confirmed that the mean frequency over three cycles approximately $5 \mathrm{~s}$ after the onset of application of the rat chow odor and that after its termination were significantly greater than the mean frequency before the odor stimulus $(P<0.05$ in both cases; paired $t$-test). The mean frequency after the onset of rat chow odor application and that after its termination were not significantly different $(P>0.5$, paired $t$-test). However, the mean frequency over three cycles approximately $5 \mathrm{~s}$ after the termination of garlic odor stimulation was significantly greater than that after stimulus onset $(P<0.01$, paired $t$-test). The mean frequencies measured after the onset of the garlic odor and after its termination were significantly greater than the mean frequency before stimulation (onset $P<0.05$, termination $P<0.001$; paired $t$-test).
Image analysis of neural activities modulated by odor stimuli

The relative change in the mean membrane potential in an area was expressed as the fractional change in the intensity of the fluorescence $(\Delta F / F)$ of the potential-sensitive dye. It should be noted that the optical signal in each pixel represents the nonlinear average of the membrane potential of several neurons and neurites within the $13 \mu \mathrm{m} \times 13 \mu \mathrm{m}$ area.

The optical signal in the cell mass, the terminal mass and the internal mass of the procerebrum oscillated (traces 2-5 in Fig. 3). The timing of the peaks of the net depolarization was found to be identical along the dorsal-ventral axis of the procerebrum when observed from the posterior side in another experiment (data not shown, see also Kimura et al., 1998b), and there was a phase delay along the distal-proximal axis (traces 2 and 3 in Fig. 3; see also Fig. 4A), as described previously in this species (Kawahara et al., 1997; Kimura et al., 1998b) and in Limax maximus (Kleinfeld et al., 1994). Because of this phase delay of the potential oscillation, it seems that an excitation occurring spontaneously in the distal area propagates repeatedly to the proximal area of the procerebrum. In addition to the procerebrum, the tentacle nerve also showed a weak oscillation in synchrony with the procerebral oscillation (trace 1 in Fig. 3), as reported previously (Kawahara et al., 1997).

When the odor of rat chow was applied for $15 \mathrm{~s}$ to the superior tentacular nose, the optical signal in the superior tentacle nerve decreased for $2 \mathrm{~s}$, indicating a net depolarization, and then returned to baseline (trace 1 in Fig. 3A). This activity pattern probably reflects the excitation and adaptation of the afferent activities of the olfactory sensory neurons. In the procerebrum, the basal level of the oscillation shifted towards depolarization (traces $2-5$ in Fig. 3A) in parallel with the excitation of the tentacle nerve. This basal depolarization was most evident in the terminal mass (trace 4 in Fig. 3A) to which the superior tentacle nerve projects. Since the cell mass also showed a basal depolarization (traces 2 and 3 in Fig. 3A), the 
A
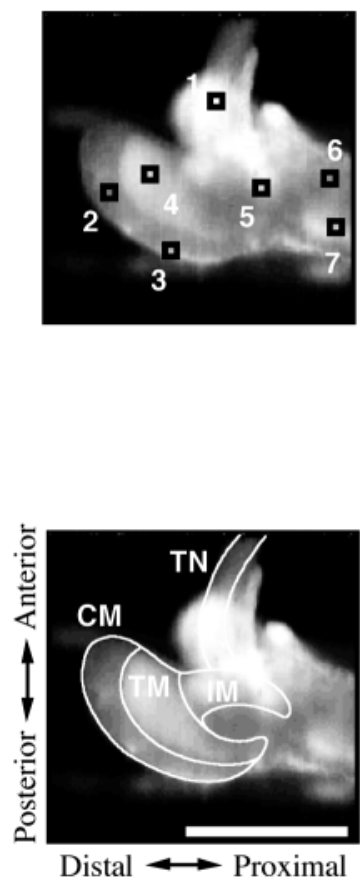

5

6

7
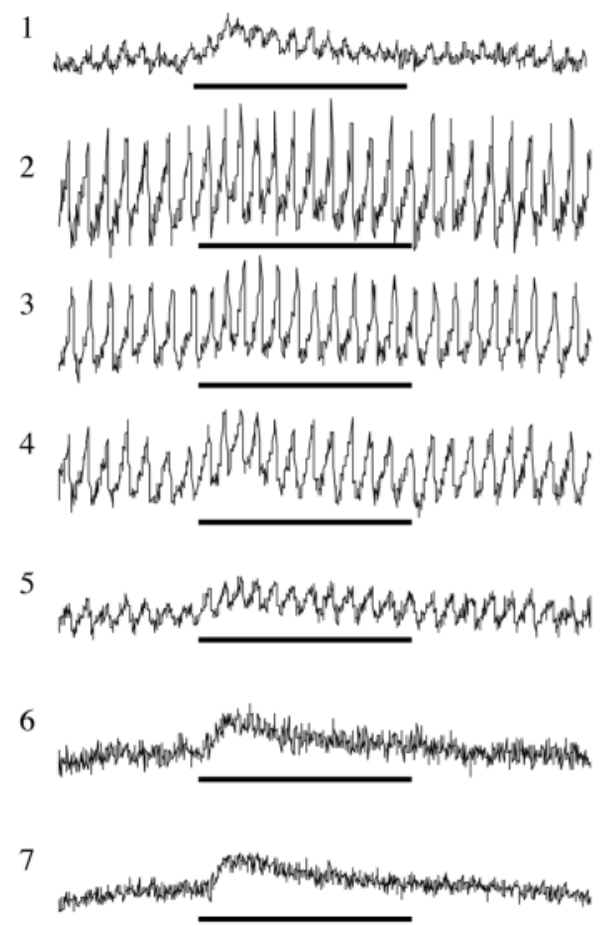

B

1

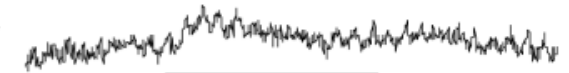

2

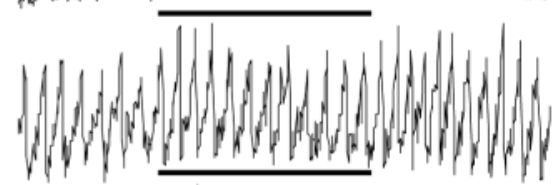

3

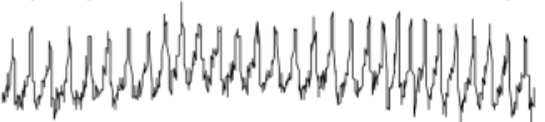

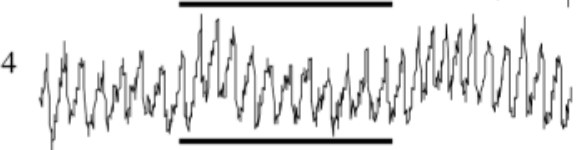

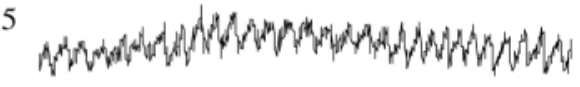

6

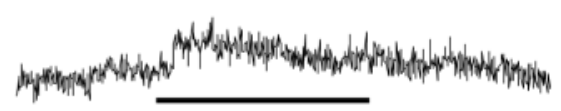

7

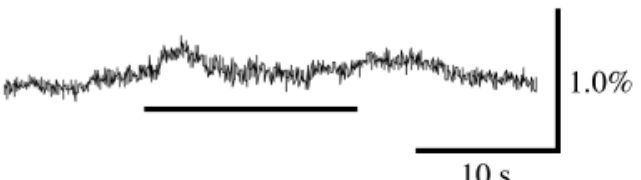

Fig. 3. Changes in the intensities of the fluorescence of the potential-sensitive dye during application of the odor of rat chow (A) or garlic (B). The fractional change in the fluorescence intensity $(-\Delta F / F)$ was obtained from $3 \times 3$ pixels in each area: 1 , tentacle nerve; 2 , distal region of the cell mass; 3, proximal region of the cell mass; 4, terminal mass; 5, internal mass; 6 and 7, regions next to the procerebrum. An upward deflection indicates net depolarization. The optical data were acquired from the preparation used for Fig. 1, and the period of the recording is indicated by the open circles in Fig. 1C,D. The bar under each trace indicates the duration of odor application. The scale bar in the fluorescence image is $400 \mu \mathrm{m}$. TN, tentacle nerve; CM, cell mass; TM, terminal mass; IM, internal mass.

depolarization in the terminal mass may reflect activation of the procerebral neurons as well as activation of the synaptic terminals of the superior tentacle nerve. Regions in the neighboring metacerebrum and mesocerebrum, which did not show oscillatory activity, also showed a net depolarization upon stimulation with the rat chow odor (traces 6 and 7 in Fig. 3A).

Fig. 3B shows the response to stimulation with garlic odor. The optical signals in the superior tentacle nerve indicated a depolarization for the first $2 \mathrm{~s}$ and then returned to baseline (trace 1 in Fig. 3B), a similar response to that observed with the rat chow odor. The basal level of the oscillating membrane potentials in the procerebrum also shifted towards depolarization (traces 2-5 in Fig. 3B). In contrast to the response to the rat chow odor, however, the basal level of oscillation in the procerebrum again shifted towards depolarization upon the termination of the odor stimulus. This depolarization corresponded to the timing of the second increase in the oscillatory frequency (see Fig. 1D) and was most evident in the terminal mass (trace 4 in Fig. 3B). Regions outside the procerebrum did not show depolarization (trace 6 in Fig. 3B) or showed a much smaller depolarization (trace 7 in Fig. 3B) after the termination of the odor application, although an initial depolarizing response to the odor application was evident.

\section{Modulation of wave propagation by odor stimuli}

To evaluate the effect of odor stimulation on wave propagation across the procerebrum, the time lag between oscillation in the proximal area of the procerebrum and that in the distal area was calculated (Fig. 4A,B). The oscillation of the fractional change in the intensity of fluorescence was obtained from $5 \times 5$ pixels (approximately $63 \mu \mathrm{m} \times 63 \mu \mathrm{m}$ ) in the distal and proximal areas, sited approximately $300 \mu \mathrm{m}$ (approximately $80 \%$ of the length of the procerebrum) apart. Since the optical signals did not always have a signal-to-noise ratio good enough to calculate the time interval between the depolarization peaks of the two traces, the time lag was defined as the time interval between the half-peak points during the hyperpolarizing phase. The mean time lag during spontaneous oscillation was $0.33 \pm 0.15 \mathrm{~s}$ before the application of the odor of rat chow (mean \pm S.D.; nine trials in seven preparations) and $0.32 \pm 0.12 \mathrm{~s}$ before the application of the odor of garlic (mean \pm S.D.; 10 trials in seven preparations), values that are approximately $15-30 \%$ of the period of the oscillation. The speed of wave propagation during spontaneous oscillation, calculated from the time lag and the distance between the centers of the measured areas, was $1.2 \pm 0.6 \mu \mathrm{m} \mathrm{ms}^{-1}$ before the application of rat chow odor and $1.2 \pm 0.5 \mu \mathrm{m} \mathrm{ms}^{-1}$ before the application of garlic odor. 
Fig. 4. Effect of odor on wave propagation from the distal to the proximal region of the procerebrum. (A) Definition of the time lag between the oscillation in the proximal region and that in the distal region. The black and red lines are traces of the fractional change in the intensity of the fluorescence $(-\Delta F / F)$ obtained from an area of $5 \times 5$ pixels in the distal (black square in B) and proximal (red square in B) regions. In each trace, the median between the peak of depolarization and the following trough of hyperpolarization was determined. The time lag was defined as the time interval between the median points during the hyperpolarizing phase of the two traces. (B) The distal region (black square) and proximal region (red square) from which optical signals were obtained. Scale bar, $400 \mu \mathrm{m}$. (C-F) Effects of the odor of rat chow $(\mathrm{C}, \mathrm{E})$ and garlic $(\mathrm{D}, \mathrm{F})$ on the time lag of oscillation (filled circle). Open circles indicate the instantaneous frequency of the local field potential (the same data as in Fig. 1C-F). The odor was applied to the superior tentacular nose during the period indicated by a bar.
A

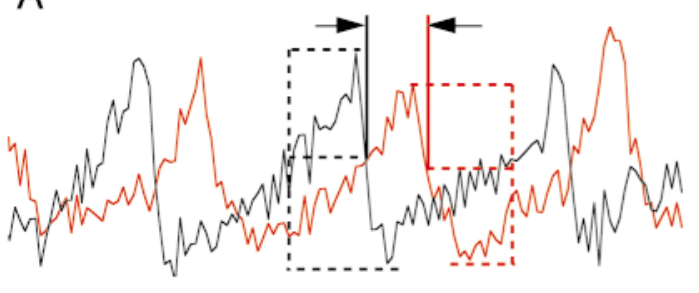

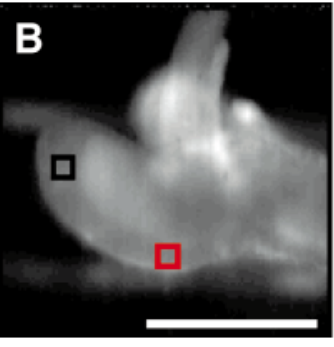
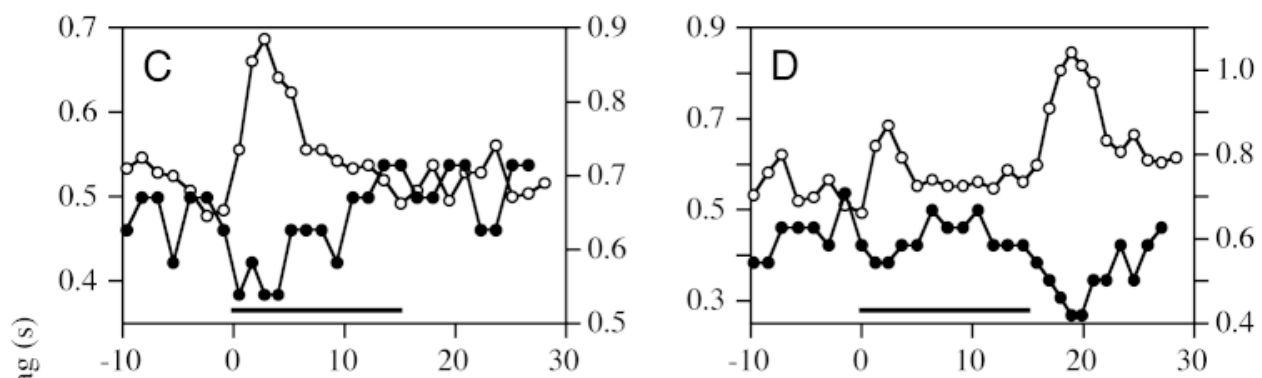

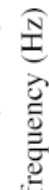
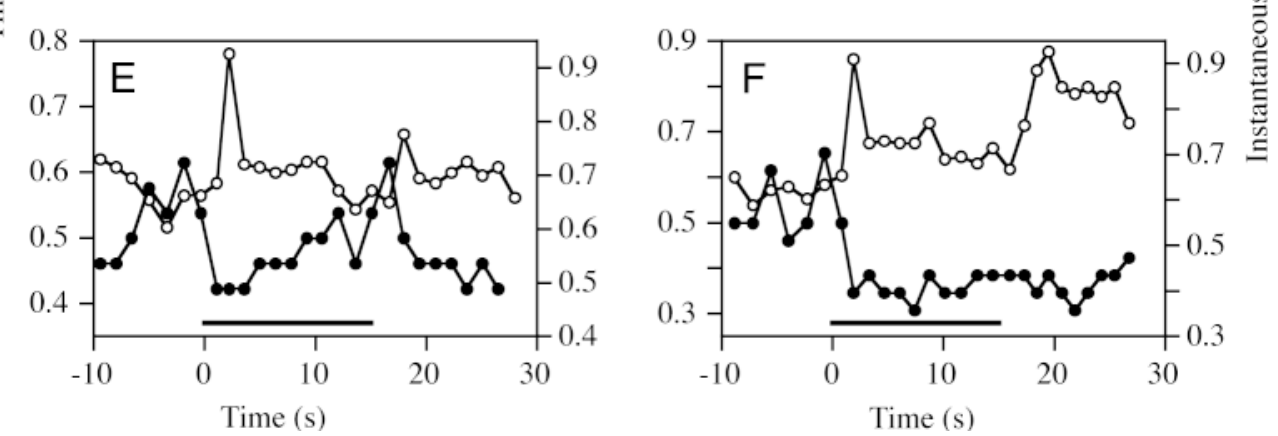

Fig. 4C shows the effects of the rat chow odor on the time lag and on the instantaneous frequency of the oscillation. Application of the odor of rat chow to the superior tentacular nose resulted in a decrease in the time lag of the proximal area relative to the distal area, indicating that wave propagation was faster than before stimulation. The decrease lasted for 3-4 cycles, and the time lag then recovered to its original level. This change was also seen in the next rat chow odor stimulation in the same preparation (Fig. 4E). Fig. 4D shows the effects of the odor of garlic on the time lag and the instantaneous
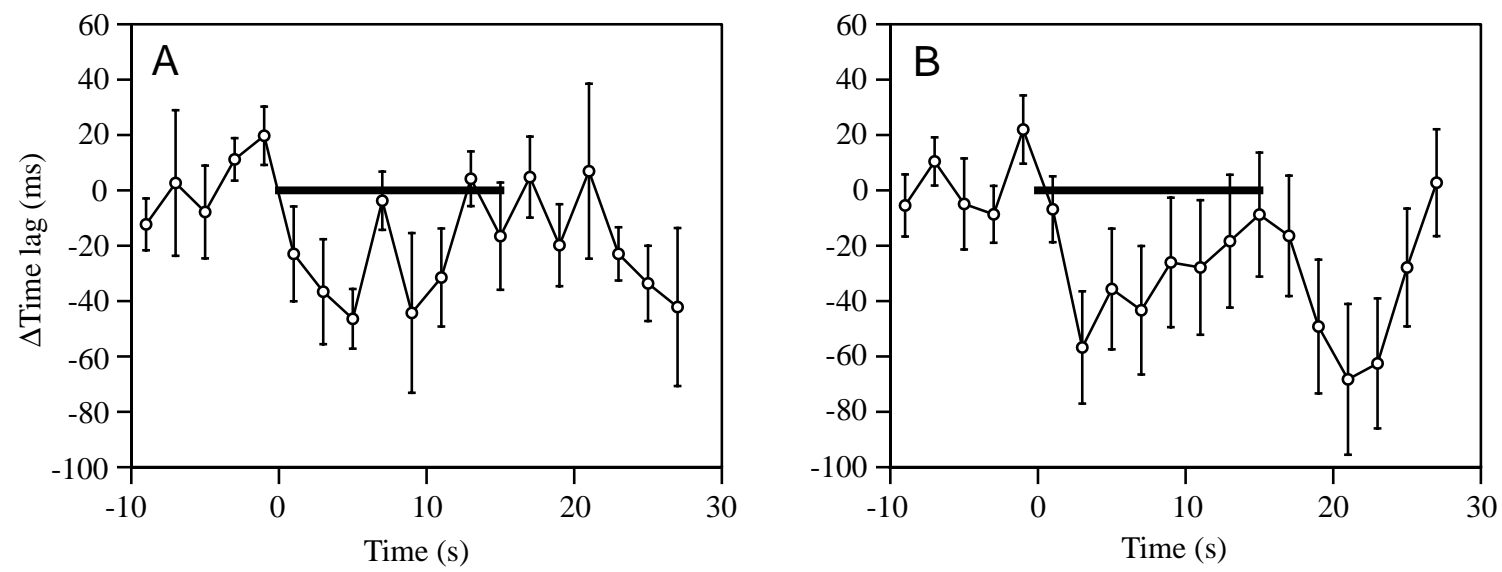

Fig. 5. Averaged data showing the effects of odor application on wave propagation in different preparations. (A) Rat chow odor (nine trials in seven preparations); (B) garlic odor (10 trials in seven preparations). The ordinate shows the change in the time lag of oscillation normalized to the mean of this value before odor stimulation. The data from different preparations were binned into $2 \mathrm{~s}$ intervals and averaged. Error bars show the standard errors of the mean (S.E.M.). The period of application of the odor is indicated by a bar. 
frequency of the oscillation. When the nose was stimulated by the odor of garlic, the time lag decreased slightly and then recovered to its original level, in a manner similar to the response to the rat chow odor. At the end of odor stimulation, the time lag decreased again, this time more strongly, in parallel with the change in the instantaneous frequency of the oscillation. A decrease in the time lag was also observed in response to a further application of the garlic odor (Fig. 4F). However, in this response, the decreased time lag was sustained during and after the odor stimulation and did not recover by the end of the optical measurement.

Fig. 5 shows the averaged data for the change in the phase delay of the oscillation in the proximal region relative to that in the distal region of the procerebrum obtained from a number of preparations stimulated with the odor of rat chow or garlic. The differential time lag ( $\Delta$ time lag) in each preparation was calculated by subtracting the time lag averaged before odor stimulation from the time lag in each cycle, binned into $2 \mathrm{~s}$ intervals. The cumulative data showed that the change in the time lag in response to the garlic odor had a second peak (Fig. 5B), while the response to the rat chow odor did not (Fig. 5A). The occurrence and the timing of these responses were in accordance with the responses observed in the oscillatory frequency. However, the relative magnitude of the responses was not the same for wave propagation and for instantaneous frequency. The second peak in the time lag was
A
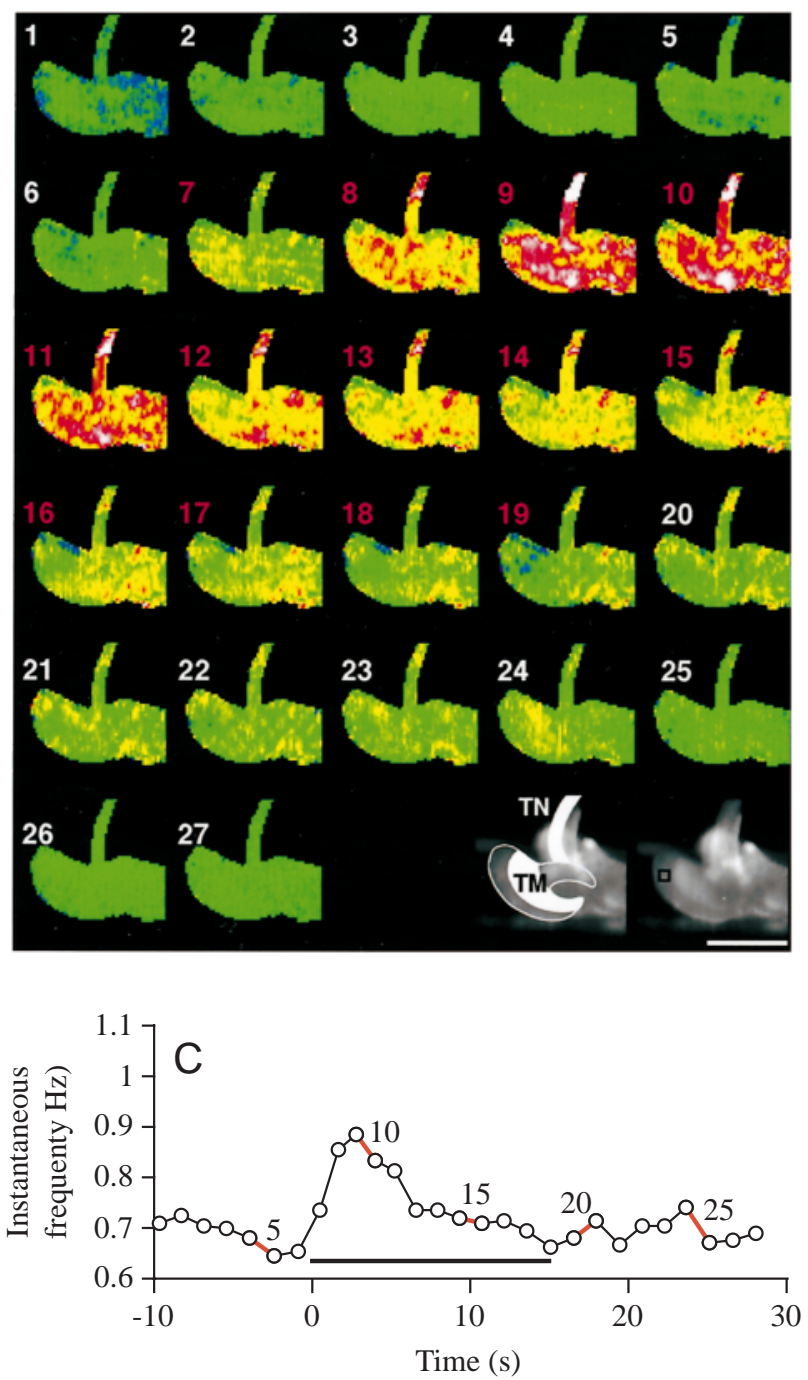

B

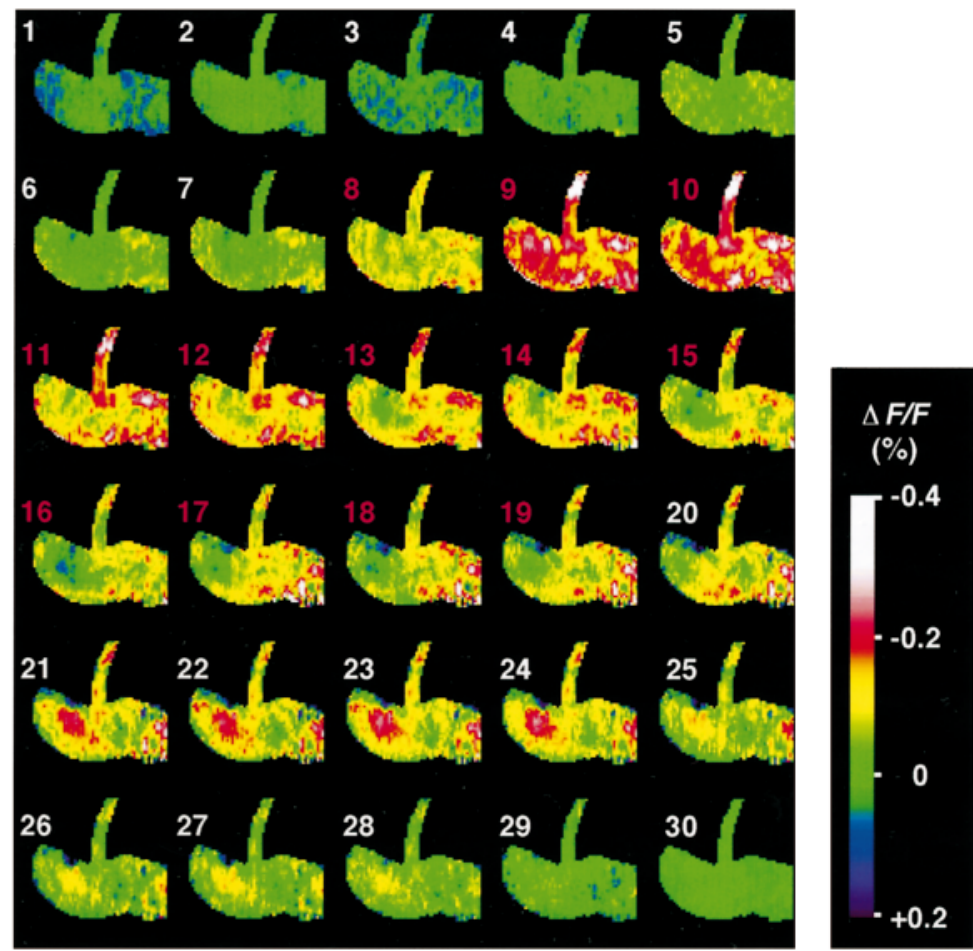

Fig. 6. Effects of odor stimulation on the mean level of net depolarization. (A,B) Fractional changes in the intensities of the fluorescence $(\Delta F / F)$ were averaged cycle-by-cycle (see Materials and methods) and pseudo-colored, as indicated by the color scale. The number indicated on each frame is the sequential cycle number during the optical recording. During the period indicated by the frames numbered in red, the preparation was stimulated with the odor of rat chow (A) or garlic (B). The last frame in A is the fluorescence image of the preparation. The black square in the image indicates the area whose peaks of depolarization were used for the cycle-by-cycle averaging. Scale bar, $400 \mu \mathrm{m}$. TN, tentacular nerve; TM, terminal mass. (C,D) The instantaneous frequency of the local field potential during the optical measurements that appear in A and B, respectively. The numbers shown beside the red lines indicate the corresponding frame numbers in A and B. These data are the same as those that appear in Fig. 1C,D, respectively. 


\section{S. TODA, S. KaWAHARA AND Y. KIRINO}

of approximately the same magnitude as the initial response, while the second peak was much bigger than the initial response for frequency (see Fig. 2B).

Statistical analysis showed that the mean of the proximal time lag over three cycles around the peak of the frequency increase during the odor stimulus and after its termination was significantly smaller than that before odor stimulation (rat chow odor, during $P<0.01$, after $P<0.05$; garlic odor, during $P<0.05$, after $P<0.01$ ). There were no significant differences between the three-peak-mean time lag during odor stimulus and that after termination of the stimulus (rat chow odor $P>0.5$, garlic odor $P>0.1$ ).

\section{Mean level of neural activity and parametric changes in the oscillation}

A cycle-by-cycle average of the optical signal was calculated to minimize the contribution of oscillatory activity. In Fig. 6A, each frame of the images represents the spatial pattern of optical signals averaged over one cycle of the oscillation. The odor of rat chow was applied during the period from frame 7 to frame 19, or between the seventh and twentieth peak of the local field potential shown in Fig. 6C. During the initial period of odor application (frames 8-15), a transient depolarization was observed in the tentacle nerve and in the terminal mass, which receives the axons of the tentacle nerve, in parallel with the change in the instantaneous frequency of the synchronous oscillation of the procerebral neurons (Fig. 6C). The neighboring regions in the metacerebrum and mesocerebrum also showed a concomitant depolarization. In Fig. 6B, garlic odor was applied during the period represented by frames $8-19$. The terminal mass depolarized transiently (frames 9 and 10) and then
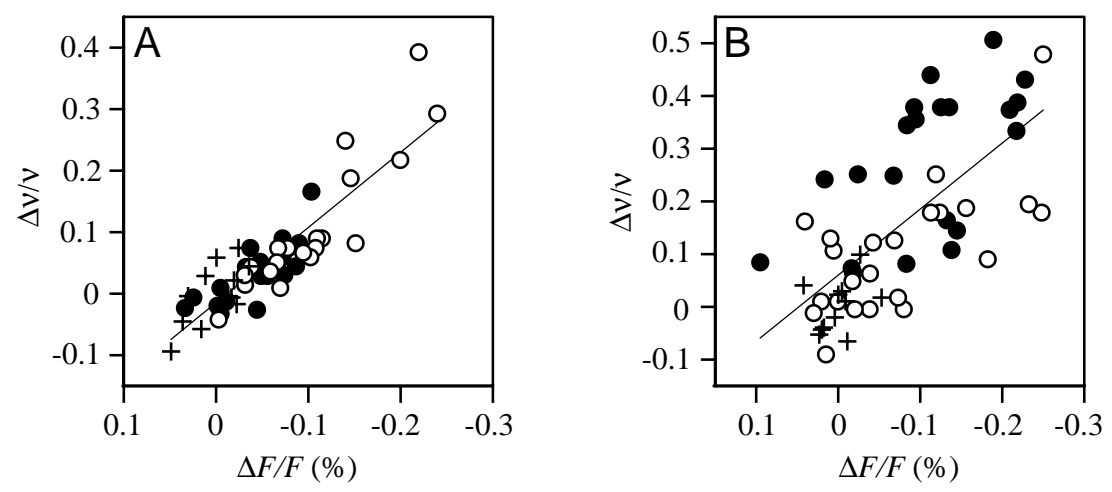

Fig. 7. Correlation between the net depolarization in the terminal mass $(\Delta F / F)$, the change in the frequency of the oscillation $(\Delta v / v)$ and the distalto-proximal time lag. (A,C,E) Rat chow odor. These graphs were constructed from two sets of data corresponding to Fig. 4C,E. (B,D,F) Garlic odor. These graphs were constructed from two sets of data corresponding to Fig. 4D,F. Plus signs, before odor application; open circles, during the odor stimulation; filled circles, after the odor stimulation. The cycle-by-cycle averaged fractional change in the fluorescence intensities $(-\Delta F / F)$ were obtained from an area of $5 \times 5$ pixels in the terminal mass. Linear regression analyses yielded the following significant relationships: (A) $y=-1.22 x-0.02 \quad(r=0.88, \quad N=51, \quad P<0.001)$; (B) $y=-1.25 x-0.06 \quad(r=0.69, \quad N=55, \quad P<0.001)$;

(C) $y=547 x+5 \quad(r=0.60, \quad N=51, \quad P<0.001)$;

(D) $y=447 x-59 \quad(r=0.44, \quad N=55, \quad P<0.001)$;

(E) $y=-358 x-6 \quad(r=0.54, \quad N=51, \quad P<0.001)$; (F) $y=-427 x-27(r=0.75, N=55, P<0.001)$.
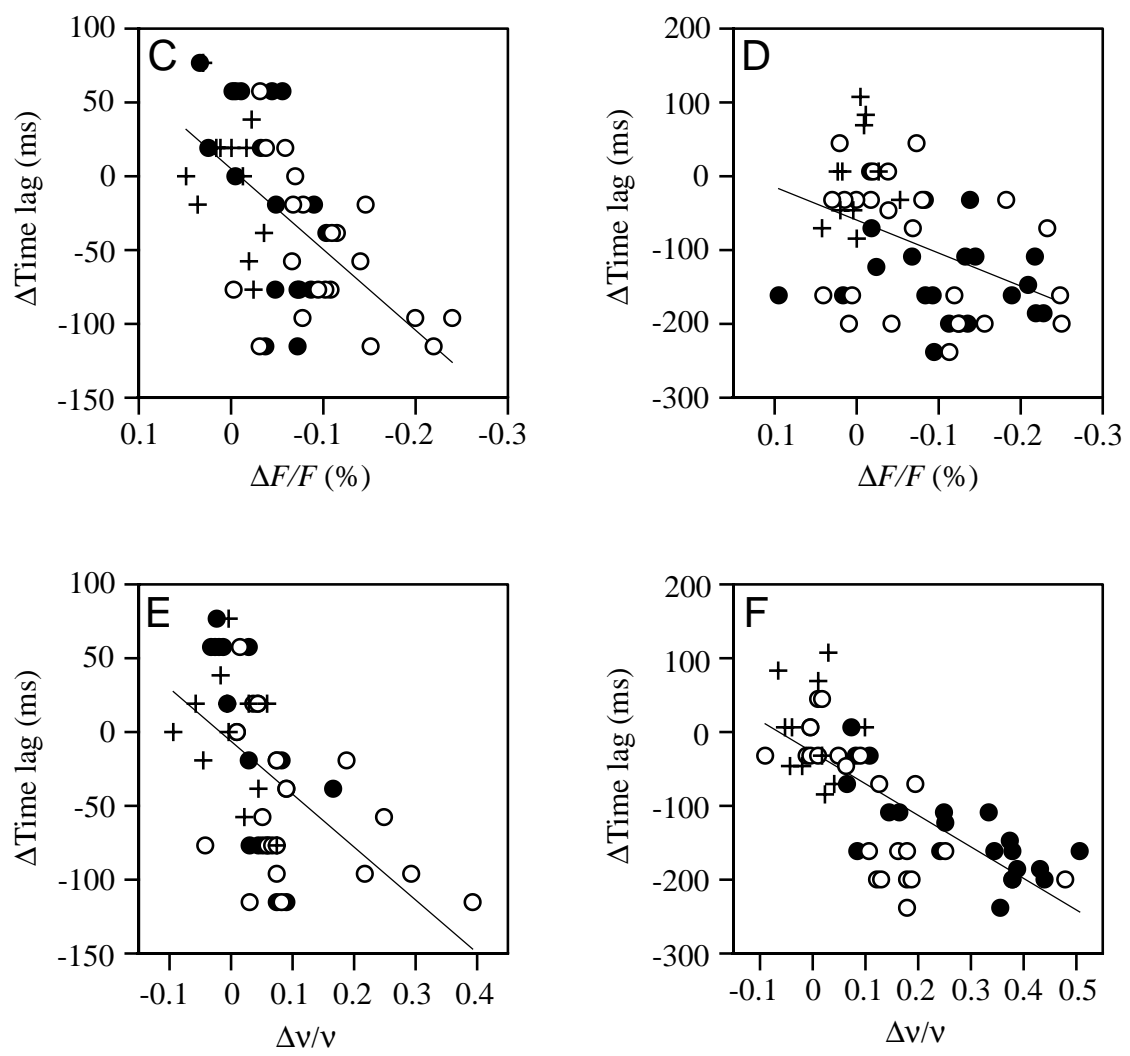
repolarized rapidly in parallel with the change in the frequency of the oscillation, while the neighboring regions remained activated. This accords with the lower basal level of the oscillation shown in trace 4 of Fig. 3B. After the termination of the odor of garlic, a depolarization occurred in the terminal mass (frames 21-24).

Fig. 7 shows the correlation between the net depolarization in the terminal mass and changes in the frequency of the oscillation (Fig. 7A,B), the correlation between the net depolarization and the proximal time lag (Fig. 7C,D) and the correlation between the frequency of oscillation and the proximal time lag (Fig. 7E,F). Both the oscillation frequency and the proximal time lag showed a significant correlation with the net depolarization in the terminal mass. However, the correlation coefficient for stimulation with the garlic odor was slightly smaller than that for stimulation with the rat chow odor. This is apparently because the fractional change in the intensity of the fluorescence was underestimated in the case of stimulation with the garlic odor using the present method of baseline correction (see Materials and methods) because the off-response was not completely finished at the end of the optical measurement (see Fig. 1D,F). The correlation between the oscillation frequency and the proximal time lag for stimulation with the garlic odor was significant and similar to that for stimulation with the rat chow odor (Fig. 7E,F).

Cumulative data from seven preparations also showed a significant correlation between the net depolarization in the terminal mass and the oscillatory frequency (Fig. 8A,B), between the net depolarization in the terminal mass and the proximal time lag (Fig. 8C,D) and between the oscillation frequency and the proximal time lag (Fig. 8E,F).
Fig. 8. Accumulated data showing the correlation between the net depolarization in the terminal mass $(\Delta F / F)$, the change in the frequency of the oscillation $(\Delta v / v)$ and the distal-to-proximal time lag in different preparations. (A,C,E) Rat chow odor (nine trials in seven preparations); $(\mathrm{B}, \mathrm{D}, \mathrm{F})$ garlic odor $(10$ trials in seven preparations). Plus signs, before odor application; open circles, during the odor stimulation; filled circles, after the odor stimulation. The cycleby-cycle averaged fractional change in the fluorescence intensities $(-\Delta F / F)$ were obtained from an area of $5 \times 5$ pixels in the terminal mass. Linear regression analyses yielded the following significant relationships:

(A) $y=-0.72 x+0.05 \quad(r=0.46, N=219, P<0.001)$;

(B) $y=-1.03 x+0.06 \quad(r=0.50, N=255, P<0.001)$;

(C) $y=223 x-13 \quad(r=0.27, \quad N=219, \quad P<0.001)$;

(D) $y=299 x-16 \quad(r=0.27, \quad N=255, \quad P<0.001)$;

(E) $y=-138 x-10 \quad(r=0.26, \quad N=219, \quad P<0.001)$;

(F) $y=-252 x-3(r=0.47, N=255, P<0.001)$.
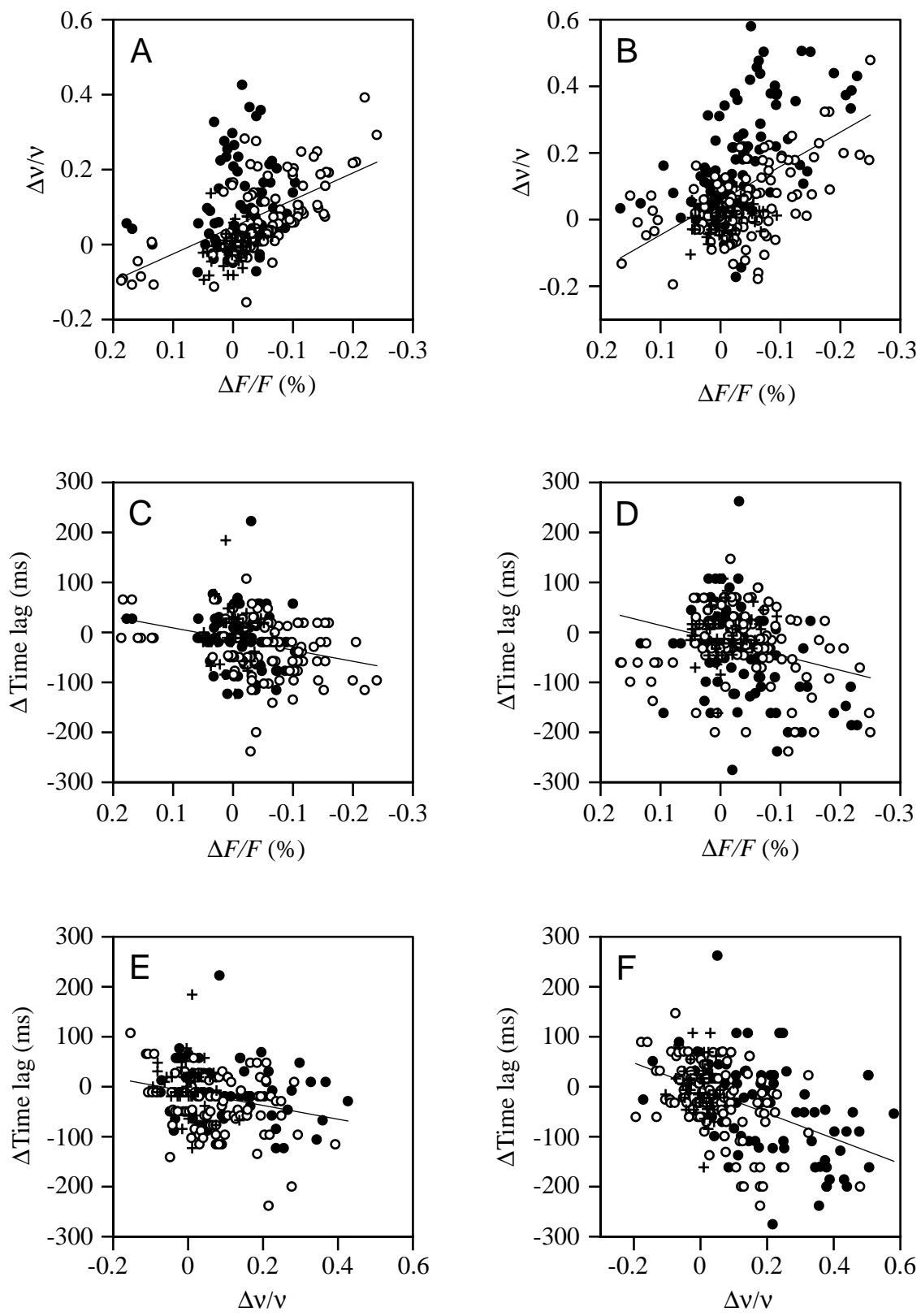


\section{Discussion}

The macroscopic neural activities of the procerebrum, which is the center for olfaction and the putative locus for the formation and storage of memory in the terrestrial slug, were investigated in Limax marginatus using optical recording techniques. To minimize mixing of the optical signals from the somatic layer (cell mass) and from the neuropil layers, of which the latter are stained more heavily than the former (see the last frame in Fig. 6A), the preparations were measured in the dorsal view (Fig. 3). Although this orientation limits the observation of spatiotemporal patterns organized over the somatic layer, it is useful for investigating the activities in the neuritic and somatic layers simultaneously (Kawahara et al., 1997). In the case of stimulation of the superior tentacle nerve, which projects extensively into the terminal mass, the strong excitation induced in the terminal mass could have obscured the optical signals originating from the cell mass if recordings had been made from a posterior view, where the cell mass overlaps with the neuropil.

In response to odor stimulation to the superior tentacular nose, the instantaneous frequency of the procerebral oscillation increased for both rat chow and garlic odors (Fig. 1). A decrease in the oscillatory frequency was rarely observed with the naturally aversive odor of garlic, which contrasts with the case of stimulation of the inferior tentacular nose, where a frequency decrease was observed in response to aversive odors (Kimura et al., 1998c). In addition to the initial increase, the garlic odor induced an off-response, i.e. a second increase in oscillation frequency after the termination of stimulation. The rat chow odor produced little or no off-response. The off-response was also observed upon application of the attractive odor of 2-ethyl-3methoxypyrazine (EMOP) as well as the aversive odor of amyl acetate to the slug Limax maximus (Gervais et al., 1996). The off-response of the procerebral oscillation may not, therefore, indicate an odor preference although, in general, an off-response is observed after the end of an inhibitory process. Odor stimulation might activate two kinds of neural activities having excitatory and inhibitory effects on the procerebral oscillation. The hyperpolarization measured optically in the terminal mass (Fig. 6B) seemed to indicate some kind of inhibitory process, although this phenomenon might merely indicate adaptation or depression in response to a prolonged odor stimulus.

Since a small area dissected from the procerebrum also showed oscillatory activity, and the wave could propagate from the proximal region to the distal region under artificial conditions (Kleinfeld et al., 1994), the macroscopic oscillation is assumed to be the product of mutual entrainment of distributed nonlinear oscillators. Therefore, like the chemical oscillation of the Belousov-Zhabotinski reaction (Nicolis and Prigogine, 1977), the frequency of the oscillation and the velocity of wave propagation may depend on closely related but different parameters. When stimulated by an odor, both the oscillatory frequency and the velocity of wave propagation changed (Fig. 4). The proximal time lag had a tendency to become smaller as the frequency of the oscillation increased (Fig. 4), but these changes were not tightly coupled. In Fig. 4F, the change in oscillatory frequency had two peaks in response to garlic odor, an initial increase and an off-response, while the proximal time lag decreased initially and then remained shortened. The accumulated data from different preparations also showed that the change in proximal time lag for the offresponse was the same as that for the initial response (Fig. 5B), in contrast to the change in the oscillatory frequency, whose off-response was larger than the initial response (Fig. 2B). The discrepancy between the responses observed in the frequency of the oscillation and in the velocity of wave propagation was also observed in Limax maximus, in which the proximal phase delay virtually disappeared in response to EMOP application even when the oscillatory frequency was almost unchanged (Delaney et al., 1994).

Changes in the frequency of the oscillation and the velocity of wave propagation were correlated with the net depolarization in the terminal mass in preparations stimulated with the odor of rat chow and garlic (Figs 7, 8). Since only the nonbursting neurons project to the terminal mass, while the neurites of the bursting neurons run mainly within the cell layer (Watanabe et al., 1998), the depolarization in the terminal mass during the off-response may represent the activity of the neurites of the nonbursting neurons rather than the activity of the bursting neurons. In addition to the neurites of the nonbursting neurons, those of extrinsic neurons (Chase and Tolloczko, 1989; Gelperin and Flores, 1997) can also contribute to the depolarization. On the other hand, the terminal mass was reported to have an oscillatory activity that is uncoupled from the procerebral oscillation by gustatory stimulation or by application of neuromodulatory substances such as serotonin (Iwama et al., 1997). In the terminal mass, therefore, there might be local microcircuits constructed from the nonbursting neurons and other extrinsic neurons, whose activities modulate the frequency of the oscillation and the velocity of wave propagation conveyed by the bursting neurons in the cell layer of the procerebrum. An involvement of the terminal mass in wave propagation in the cell layer has also been indicated by experiments in which a segment of the cell layer is ablated and the wave still propagates through the ablation, via the terminal mass layer (Ermentrout et al., 1998).

In the present study, a spatial pattern specific to an odor could not be detected, at least in the dorsal view of the procerebrum. Since the terminal mass receives abundant projections from the superior tentacle nerve, it might be difficult to detect an odorspecific or learning-specific pattern in the posterior view, as detected with stimulation of the inferior tentacle nerve (Kimura et al., 1998b) whose projection to the terminal mass was much sparser than that of the superior tentacle nerve. For this purpose, a more selective staining of the cell mass would be needed using a potential-sensitive dye or a $\mathrm{Ca}^{2+}$-sensitive dye. The latter would be expected to report excitation of the cell bodies, which have a much larger amount of cytoplasm than the neurites, when used in bath application (Inoue et al., 1998).

This work was supported by Grants-in-Aid for Scientific Research from the Ministry of Education, Science, Sports and 
Culture, Japan (Nos 10480176, 08680714, 07557143 and 07279105), and by a Grant from the Program for Promotion of Basic Research Activities for Innovative Biosciences, Japan.

\section{References}

Brierley, M. J., Yeoman, M. S. and Benjamin, P. R. (1997). Glutamatergic N2v cells are central pattern generator interneurons of the Lymnaea feeding system: new model for rhythm generation. J. Neurophysiol. 78, 3396-3407.

Castelo-Branco, M., Neuenschwander, S. and Singer, W. (1998). Synchronization of visual responses between the cortex, lateral geniculate nucleus and retina in the anesthetized cat. J. Neurosci. 18, 6395-6410.

Chase, R. (1985). Responses to odors mapped in snail tentacles and brain by $\left[{ }^{14} \mathrm{C}\right]$-2-deoxyglucose autoradiography. J. Neurosci. 5, 2930-2939.

Chase, R. and Tolloczko, B. (1989). Interganglionic dendrites constitute an output pathway from the procerebrum of the snail Achatina fulica. J. Comp. Neurol. 283, 143-152.

Degtyarenko, A. M., Simon, E. S. and Burke, R. E. (1996). Differential modulation of disynaptic cutaneous inhibition and excitation in ankle flexor motoneurons during fictive locomotion. J. Neurophysiol. 76, 2972-2985.

Delaney, K. R., Gelperin, A., Fee, M. S., Flores, J. A., Gervais, R., Tank, D. W. and Kleinfeld, D. (1994). Waves and stimulusmodulated dynamics in an oscillating olfactory network. Proc. Natl. Acad. Sci. USA 91, 669-673.

Ermentrout, B., Flores, J. and Gelperin, A. (1998). Minimal model of oscillations and waves in the Limax olfactory lobe with tests of the model's predictive power. J. Neurophysiol. 79, 2677-2689.

Freeman, W. J. (1978). Spatial properties of an EEG event in the olfactory bulb and cortex. Electroencephalogr. Clin. Neurophysiol. 44, 586-605.

Gelperin, A. and Flores, J. (1997). Vital staining from dye-coated microprobes identifies new olfactory interneurons for optical and electrical recording. J. Neurosci. Meth. 72, 97-108.

Gelperin, A., Hopfield, J. J. and Tank, D. W. (1985). The logic of Limax learning. In Model Neural Networks and Behavior (ed. A. I. Selverston), pp. 237-261. New York: Plenum Press.

Gelperin, A. and Tank, D. W. (1990). Odour-modulated collective network oscillations of olfactory interneurons in a terrestrial mollusc. Nature 345, 437-440.

Gervais, R., Kleinfeld, D., Delaney, K. R. and Gelperin, A. (1996). Central and reflex neuronal responses elicited by odor in a terrestrial mollusk. J. Neurophysiol. 76, 1327-1339.

Grillner, S., Deliagina, T., Ekeberg, Ö., el Manira, A., Hill, R. H., Lansner, A., Orlovsky, G. N. and Wallén, P. (1995). Neural networks that co-ordinate locomotion and body orientation in lamprey. Trends Neurosci. 18, 270-279.

Inoue, T., Kawahara, S., Toda, S., Watanabe, S. and Kirino, Y. (1998). Selective optical recording of the neural activity in the olfactory center of land slug using a calcium indicator dye. Bioimages 6, 59-67.
Iwama, A., Kimura, T., Yamada, A., Kono, E., Suzuki, H. and Sekiguchi, T. (1997). A novel kind of oscillation originating from the terminal mass of the procerebral lobe in a terrestrial mollusc. Soc. Neurosci. Abstr. 23, 1342.

Kawahara, S., Toda, S., Suzuki, Y., Watanabe, S. and Kirino, Y. (1997). Comparative study on neural oscillation in the procerebrum of the terrestrial slugs Incilaria bilineata and Limax marginatus. J. Exp. Biol. 200, 1851-1861.

Kawahara, S., Yano, M. and Shimizu, H. (1994). Radular mechanosensory neuron in the buccal ganglia of the terrestrial slug, Incilaria fruhstorferi. J. Comp. Physiol. A 174, 111-120.

Kimura, T., Suzuki, T., Kono, E. and Sekiguchi, T. (1998a). Mapping of interneurons that contribute to food aversive conditioning in the slug brain. Learn. Memory 4, 376-388.

Kimura, T., Toda, S., Sekiguchi, T., Kawahara, S. and Kirino, Y. (1998b). Optical recording analysis of olfactory response of the procerebral lobe in the slug brain. Learn. Memory 4, 389-400.

Kimura, T., Toda, S., Sekiguchi, T. and Kirino, Y. (1998c). Behavioral modulation induced by food odor aversive conditioning and its influence on the olfactory responses of an oscillatory brain network in the slug Limax marginatus. Learn. Memory 4, 365-375.

Kleinfeld, D., Delaney, K. R., Fee, M. S., Flores, J. A., Tank, D. W. and Gelperin, A. (1994). Dynamics of propagating waves in the olfactory network of a terrestrial mollusc: an electrical and optical study. J. Neurophysiol. 72, 1402-1419.

Laurent, G. and Naraghi, M. (1994). Odorant-induced oscillations in the mushroom bodies of the locust. J. Neurosci. 14, 2993-3004.

Nicolis, G. and Prigogine, I. (1977). Self-organization in Nonequilibrium Systems. From Dissipative Structure to Order through Fluctuations. New York: John Wiley \& Sons.

Ritz, R. and Sejnowski, T. J. (1997). Synchronous oscillatory activity in sensory systems: new vistas on mechanisms. Curr. Opin. Neurobiol. 7, 536-546.

Sahley, C., Rudy, J. W. and Gelperin, A. (1981). An analysis of associative learning in a terrestrial mollusc: higher-order conditioning, blocking and a transient US pre-exposure effect. $J$. Comp. Physiol. A 144, 1-8.

Schaffer, P., Ahammer, H., Müller, W., Koidl, B. and Windisch, H. (1994). Di-4-ANEPPS causes photodynamic damage to isolated cardiomyocytes. Pflügers Arch. 426, 548-551

Sekiguchi, T., Yamada, A. and Suzuki, H. (1997). Reactivationdependent changes in memory states in the terrestrial slug Limax flavus. Learn. Memory 4, 356-364

Vinay, L., Barthe, J. Y. and Grillner, S. (1996). Central modulation of stretch receptor neurons during fictive locomotion in lamprey. $J$. Neurophysiol. 76, 1224-1235.

Watanabe, S., Kawahara, S. and Kirino, Y. (1998). Morphological characterization of the bursting and nonbursting neurons in the olfactory center of the terrestrial slug Limax marginatus. J. Exp. Biol. 201, 925-930.

Yamada, A., Sekiguchi, T., Suzuki, H. and Mizukami, A. (1992). Behavioral analysis of internal memory states using coolinginduced retrograde amnesia in Limax flavus. J. Neurosci. 12, 729-735. 